\title{
Influence of Donor Age and Passage Number on Angiogenic Activity in Human Adipose-Derived Stem Cell-Conditioned Media
}

Takahiro Nakamura', Tomohiko Kazama², Yuki Nagaoka², Yasuji Inamo', Hideo Mugishima1, Shori Takahashi' and Taro Matsumoto*

${ }^{1}$ Department of Pediatrics, Nihon University School of Medicine, Tokyo, Japan

${ }^{2}$ Department of Functional Morphology, Division of Cell Regeneration and Transplantation, Nihon University School of Medicine, Tokyo, Japan

\begin{abstract}
Introduction: Adipose-derived stem/stromal cells (ASCs) are considered a promising cell source for therapeutic angiogenesis because the cells can be prepared following a minimally invasive procedure and because they secrete a variety of angiogenic cytokines. In the present study, the influence of donor age and passage number on angiogenic activity of ASC-conditioned media (ASC-CM) was examined.
\end{abstract}

Methods: Human ASCs (donor age, 5 months to 82 years; $n=10$ ) were cultured, and ASC-CM were collected at passages 2, 4, and 6 . The angiogenic activity of ASC-CM was evaluated with the tube formation assay using a system in which human umbilical vein endothelial cells (HUVECs) and fibroblasts were co-cultured. The concentrations of vascular endothelial growth factor-A (VEGF-A) and hepatocyte growth factor (HGF) in each ASC-CM were measured using an enzyme-linked immunosorbent assay.

Results: A donor age over 60 years affected the proliferative capacity of ASCs at passage 4 and later. ASCCM significantly enhanced HUVEC tube formation, and this response was not influenced by donor age. ASC-CM at passage 6 showed a lower tube formation capacity compared to ASC-CM at passage 4, although the capacity was still equivalent to the positive control (medium containing $10 \mathrm{ng} / \mathrm{mL}$ VEGF-A). A donor age over 26 years affected VEGF-A but not HGF levels in ASC-CM, although we found no direct correlation between VEGF-A/HGF levels and the tube formation capacity.

Conclusion: Our results demonstrate a passage number-dependent but a donor age-independent decline in the angiogenic activity of ASC-CM.

Keywords: Angiogenesis; Mesenchymal stem cells; Tube formation; Vascular endothelial growth factor; Hepatocyte growth factor

Abbreviations: ANOVA: Analysis Of Variance; Ascs: AdiposeDerived Stem/Stromal Cells; ASMA: $a$-Smooth Muscle Actin; CM: Conditioned Media; DMEM: Dulbecco's Modified Eagle's Medium; ELISA: Enzyme-Linked Immunosorbent Assay; FBS: Fetal Bovine Serum; FGF-2: Fibroblast Growth Factor-2; HGF: Hepatocyte Growth Factor; Huvecs: Human Umbilical Vein Endothelial Cells; NC: Negative Control; NO: Nitric Oxide; PBS: Phosphate-Buffered Saline; PC: Positive Control; ROS: Reactive Oxygen Species; SVF: Stromal Vascular Fraction; VEGF-A: Vascular Endothelial Growth Factor-A.

\section{Introduction}

Adipose tissue is composed of a population of multipotent progenitors, termed adipose-derived stem/stromal cells (ASCs), which can be isolated and expanded from the stromal vascular fraction (SVF) of collagenase-digested adipose tissue [1]. The phenotype and functional properties of ASCs are similar to bone marrow mesenchymal stem cells (MSCs). Cultured ASCs satisfy the minimal criteria for defining MSCs as proposed by the International Society of Cell Therapy based on their phenotype $\left(\mathrm{CD}^{2} 3^{+}, \mathrm{CD} 90^{+}, \mathrm{CD} 105^{+}, \mathrm{CD} 11 \mathrm{~b} / \mathrm{CD} 14^{-}, \mathrm{CD} 19 / \mathrm{CD}^{-} 3 \mathrm{~b}^{-}\right.$, $\mathrm{CD} 34^{-}, \mathrm{CD} 45^{-}, \mathrm{HLA}^{-} \mathrm{DR}^{-}$), their plastic-adherent properties, and their potential to differentiate into multiple lineages including adipogenic, chondrogenic, and osteogenic lineages [2]. Because adipose tissue is abundant in most individuals and can be harvested with a less invasive procedure that required for harvesting bone marrow, ASCs are thought to be a promising cell source for a variety of cell-based therapies and tissue engineering [3].

Similar to bone marrow MSCs, ASCs possess angiogenic potential in vitro and in vivo. Transplantation of ASCs improves recovery of blood flow in mice and rats following an ischemic vascular injury [4-7].
The therapeutic effect of ASCs in ischemic models is mainly mediated by the release of a number of angiogenic factors such as vascular endothelial growth factor-A (VEGF-A) and hepatocyte growth factor (HGF) $[4,6,8,9]$. ASCs can be directed to differentiate into vascular endothelial cells $[5,10-12]$ and smooth muscle-like cells $[13,14]$ and contribute to repair in ischemic tissue. ASCs also promote mobilization of bone marrow endothelial progenitors that also participate in neovascularization $[15,16]$. Several clinical trials of cell therapies using ASCs or SVF cells for cardiovascular diseases are ongoing [17].

The proliferation and differentiation potential of MSCs/ASCs is known to be affected by in vitro aging (passage number in culture) [18$21]$ and by in vivo aging (donor age) $[18,20,22-25]$. On the other hand, limited information is currently available regarding the angiogenic potential of aged ASCs [26-28]. In the setting of ASC-based therapeutic angiogenesis, clarification of how in vivo and in vitro aging of cultured human ASCs affects angiogenic potential is needed, because the population of patients with cardiovascular diseases is generally elderly,

*Corresponding author: Taro Matsumoto, Department of Functional Morphology, Division of Cell Regeneration and Transplantation, Nihon University School of Medicine, 30-1 Ohyaguchi-Kamicho, Itabashi-ku, Tokyo 173-8610, Japan, Tel: +8133972 8111 (Ext.: 2700); Fax: 8133972 8666; E-mail: matsumoto.taro@nihon-u.ac.jp

Received September 01, 2015; Accepted September 24, 2015; Published September 26, 2015

Citation: Nakamura T, Kazama T, Nagaoka $\mathrm{Y}$, Inamo $\mathrm{Y}$, Mugishima $\mathrm{H}$, et al (2015) Influence of Donor Age and Passage Number on Angiogenic Activity in Human Adipose-Derived Stem Cell-Conditioned Media. J Stem Cell Res Ther 5 307. doi:10.4172/2157-7633.1000307

Copyright: (c) 2015 Nakamura T, et al. This is an open-access article distributed under the terms of the Creative Commons Attribution License, which permits unrestricted use, distribution, and reproduction in any medium, provided the original author and source are credited. 
and culturing the cells for several passages is commonly needed to obtain sufficient numbers and purity of cells for transplantation. In the present study, we examined the influence of donor age and passage number on angiogenic activity in human ASC-conditioned media (ASC-CM). To evaluate angiogenic activities, we employed an in vitro tube formation assay that involves co-culture of human umbilical vein endothelial cells (HUVECs) with fibroblasts as stromal cells. This co-culture assay mimics in vivo capillary vessel formation and is reproducible, sensitive, and permits quantification of the angiogenic activity of substances including chemical compounds and conditioned media from cells [29]. Our results demonstrated a passage number-dependent but a donor age-independent decline in the angiogenic activity of ASC-CM.

\section{Materials and Methods}

\section{Cell preparation}

Samples of human subcutaneous adipose tissue (donor age, 5 months to 82 years; $\mathrm{n}=10$ ) were obtained from patients undergoing surgery at Nihon University Itabashi Hospital. Patients provided written informed consent, and the Ethics Committee of Nihon University School of Medicine approved the study. Preparation of cultured ASCs was done as described previously [30]. Briefly, approximately $2 \mathrm{~g}$ adipose tissue was minced and digested in $0.1 \%$ collagenase solution (Collagenase type I, Koken, Tokyo, Japan) at $37^{\circ} \mathrm{C}$ for $1 \mathrm{~h}$ with gentle agitation. After filtration and centrifugation at $135 \times g$ for $3 \mathrm{~min}$, the pellet was washed with phosphate-buffered saline (PBS) and resuspended in Dulbecco's modified Eagle's medium (DMEM, Invitrogen, Carlsbad, CA) containing 20\% fetal bovine serum (FBS, JRH Bioscience, Lenexa, KS). The cells $\left(5 \times 10^{6}\right)$ were incubated at $37^{\circ} \mathrm{C}$ in $5 \% \mathrm{CO}_{2}$ in $25-\mathrm{cm}^{2}$ culture flasks. The medium was replaced every $3-4$ days. When the cells reached $80-90 \%$ confluence after 10 to 14 days of culture, the cells were washed with PBS, detached with $0.05 \%$ trypsin-EDTA (Invitrogen), and reseeded at a density of $1 \times 10^{5}$ cells on a $100-\mathrm{mm}$ dish (passage 1 ). The cells were serially subcultured up to passage 8 at a density of $1 \times 10^{5}$ cells per 100-mm dish. The morphology of the cells at each passage was evaluated with phase contrast microscopy (Nikon Eclipse TE2000-U, Nikon, Tokyo, Japan). When the cells did not reach $70 \%$ confluence by 10 days after subculture, no further passaging was performed after confirming an increase in senescent cells in the culture. The senescent cells were detected with a senescence $\beta$-galactosidase staining kit (Cell Signaling, Beverly, MA).

\section{In vitro differentiation assay}

Differentiation assays for adipocytes, osteoblasts, chondrocytes, and smooth muscle cells were performed as previously described [30,31] with minor modifications. Briefly, for adipogenic differentiation, confluent ASCs were cultured for 14 days in 10\% FBS in DMEM supplemented with $1 \mu \mathrm{M}$ dexamethasone, $0.5 \mathrm{mM}$ 3-isobutyl-1-methylxanthine (Sigma-Aldrich, St. Louis, MO), and 5\% insulin-transferrin-selenium-X (Invitrogen). Cells were fixed with $4 \%$ paraformaldehyde and stained with Oil Red O (Sigma-Aldrich) for $20 \mathrm{~min}$. Intracellular accumulation of neutral lipids was examined with light microscopy (Nikon Eclipse TE2000-U). For osteogenic differentiation, confluent ASCs were cultured for 3 weeks in 10\% FBS in DMEM supplemented with 100 $\mathrm{nM}$ dexamethasone, $10 \mathrm{mM} \beta$-glycerophosphate, and $50 \mu \mathrm{M}$ L-ascorbic acid (Sigma-Aldrich). Cells were fixed with $4 \%$ paraformaldehyde and then stained for alkaline phosphatase using an alkaline phosphatase staining kit (Muto Pure Chemicals, Tokyo, Japan). Other cells were treated with $1 \%$ alizarin red S (Sigma-Aldrich) at room temperature for 3 min to visualize calcium precipitation. Stained cells were examined with light microscopy (Nikon Eclipse TE2000-U). For chondrogenic differentiation, cells were seeded at a density of $2 \times 10^{6}$ cells per pellet in $15-\mathrm{cm}^{3}$ conical tubes. Cells were centrifuged at $300 \times g$ for 3 min to collect cells at the bottom of each tube, facilitating the formation of a cell pellet. Cells were maintained at $37^{\circ} \mathrm{C}$ with $5 \% \mathrm{CO}_{2}$ in $\mathrm{NH}$ ChondroDiff medium (Miltenyi Biotec, Bergish Gladbach, Germany) for 21 days. The medium was replaced every 3-4 days. After photography under a stereomicroscope (VB-7000, Keyence, Osaka, Japan), the pellets were fixed, paraffin embedded, and sectioned at $5 \mu \mathrm{m}$ thickness. The sections were fixed in $4 \%$ paraformaldehyde, permeabilized in $0.2 \%$ Triton X-100, blocked with normal goat serum, and incubated with mouse monoclonal anti-human aggrecan antibody (1:100, MAB19310, Chemicon, Temecula, CA) at $4^{\circ} \mathrm{C}$ overnight, followed by Alexa 594 goat anti-mouse IgG antibody (1:500, Invitrogen). After staining the nuclei with Hoechst 33342, the samples were examined using a confocal laser scanning microscope (Olympus FluoView FV10i, Olympus, Tokyo, Japan). For smooth muscle cell differentiation, $5 \times 10^{4}$ cells were seeded on 35-mm dishes and incubated with 5\% FBS in DMEM supplemented with $5 \mathrm{ng} / \mathrm{mL}$ transforming growth factor- $\beta 1$ (R\&D Systems, Minneapolis, $\mathrm{MN}$ ) for 7 days. Cells were fixed in $4 \%$ paraformaldehyde, permeabilized in $0.2 \%$ Triton X-100, blocked with normal goat serum, and incubated with mouse monoclonal anti-human $\bigotimes$-smooth muscle actin (ASMA) antibody (1:200, DakoCytomation, Glostrup, Denmark) followed by Alexa 594 goat anti-mouse IgG antibody (1:500). After staining nuclei with Hoechst 33342, the samples were examined using an immunofluorescence microscope (Nikon Eclipse TE 2000-U).

\section{Preparation of ASC-CM}

ASCs at passages 2, 4, and 6 were cultured on 100-mm dishes until they reached confluence. The cells were then washed once with PBS, the medium was replaced with $5 \mathrm{~mL}$ of $5 \%$ FBS in DMEM, and cells were cultured for a further $72 \mathrm{~h}$ at $37^{\circ} \mathrm{C}$ in $5 \% \mathrm{CO}_{2}$ in air. Conditioned medium was collected and centrifuged at $300 \times g$ for $10 \mathrm{~min}$, and then the medium was filtered through a $0.45-\mu \mathrm{m}$ filter and cryopreserved at $-80^{\circ} \mathrm{C}$ in a deep freezer. Immediately prior to use, samples were rapidly thawed in a $37^{\circ} \mathrm{C}$ water bath.

\section{Tube formation assay}

ASC-CM-induced HUVEC tube formation capacity was evaluated using an Angiogenesis kit (Kurabo, Osaka, Japan) according to the manufacturer's instructions. HUVECs were co-cultured with human fibroblasts in 24-well plates with ASC-CM diluted 1:1 with the assay medium (Kurabo). A 1:1 mixture of 5\% FBS in DMEM and the assay medium was used as a negative control, and the negative control medium supplemented with $10 \mathrm{ng} / \mathrm{mL}$ VEGF-A (R\&D Systems) was used as a positive control. The medium was replaced every 3 days. After 11 days of culture, cells were fixed with $70 \%$ ethanol, blocked with $1 \%$ bovine serum albumin in PBS, incubated with mouse monoclonal anti-human $\mathrm{CD} 31$ antibody $(1: 4000, \mathrm{Kurabo})$ at $37^{\circ} \mathrm{C}$ for $1 \mathrm{~h}$, and then incubated with alkaline phosphatase-conjugated goat anti-mouse IgG (1:500, Kurabo) at $37^{\circ} \mathrm{C}$ for $1 \mathrm{~h}$. Immunoreactivity was visualized using nitro blue tetrazolium chloride and 5-bromo-4-chloro-3-indoly-phosphate. Stained samples were examined with light microscopy (Nikon Eclipse TE2000-U) under a $4 \times$ objective lens, and three random fields in each well were photographed. The total tube length and total tube area in three fields/well were quantified using Angiogenesis Image Analyzer software (Ver. 2.04, Kurabo). Each sample was tested in triplicate wells.

\section{Enzyme-linked immunosorbent assay (ELISA)}

The concentrations of VEGF-A, HGF, and fibroblast growth factor-2 (FGF-2) in ASC-CM were determined with ELISA according to the 
Citation: Nakamura T, Kazama T, Nagaoka Y, Inamo Y, Mugishima H, et al. (2015) Influence of Donor Age and Passage Number on Angiogenic Activity in Human Adipose-Derived Stem Cell-Conditioned Media. J Stem Cell Res Ther 5: 307. doi:10.4172/2157-7633.1000307

Page 3 of 10

manufacturer's protocol (R\&D Systems). Absorbance at $450 \mathrm{~nm}$ was read using a microplate reader (Model680XR, Bio-Rad Laboratories, Hercules, CA). Each sample was tested in triplicate wells.

\section{Statistical analysis}

All data are presented as the mean \pm SD. One-way analysis of variance (ANOVA) and Tukey's multiple comparison tests were used to compare the tube length and tube area of different donors. Friedman's ANOVA and the Wilcoxon signed ranks test were used to compare the tube length and tube area of different cell passages. The Mann-Whitney $\mathrm{U}$ test was used to compare VEGF-A levels between the groups. Statistical analysis was performed using JMP (version 8, SAS Institute Japan, Tokyo, Japan). $\mathrm{p}<0.05$ was considered statistically significant.

\section{Results}

\section{Preparation and properties of cultured human ASCs}

We first prepared cultured ASCs from SVF cells of collagenasedigested human adipose tissue. Preparation of ASCs was successful for all donors we examined $(n=10)$. Cultured ASCs at first passage exhibited spindle-shaped morphology with high proliferative activity (Figure 1A). The cells showed the ability to differentiate into adipocytes (Figure 1B), osteoblasts (Figure 1C and D), chondrocytes (Figure 1E and F), and smooth muscle-like cells (Figure 1G) when they were cultured in appropriate culture conditions. Immunophenotypic analysis revealed that ASCs expressed stromal cell markers for CD73, CD90, and CD105, and did not express the hematopoietic markers CD45, CD34, CD14, CD19, and HLA-DR (data not shown). These immunophenotypic and multilineage differentiation properties of the cells corresponded to the minimum definition of ASCs as described previously [2].

Donor age- and passage number-dependent changes in morphology and proliferative capacity of ASCs

ASCs obtained from each donor were subcultured, and changes in cell morphology and proliferative capacity were examined. Up to passage 3, all ASC cultures exhibited spindle-shaped morphology and high proliferative capacity with a population doubling time of $\leq 72 \mathrm{~h}$. Thus, cells reached confluence within 7 days after seeding. At passage 4, ASCs from donors aged 60 years and 82 years became enlarged and showed
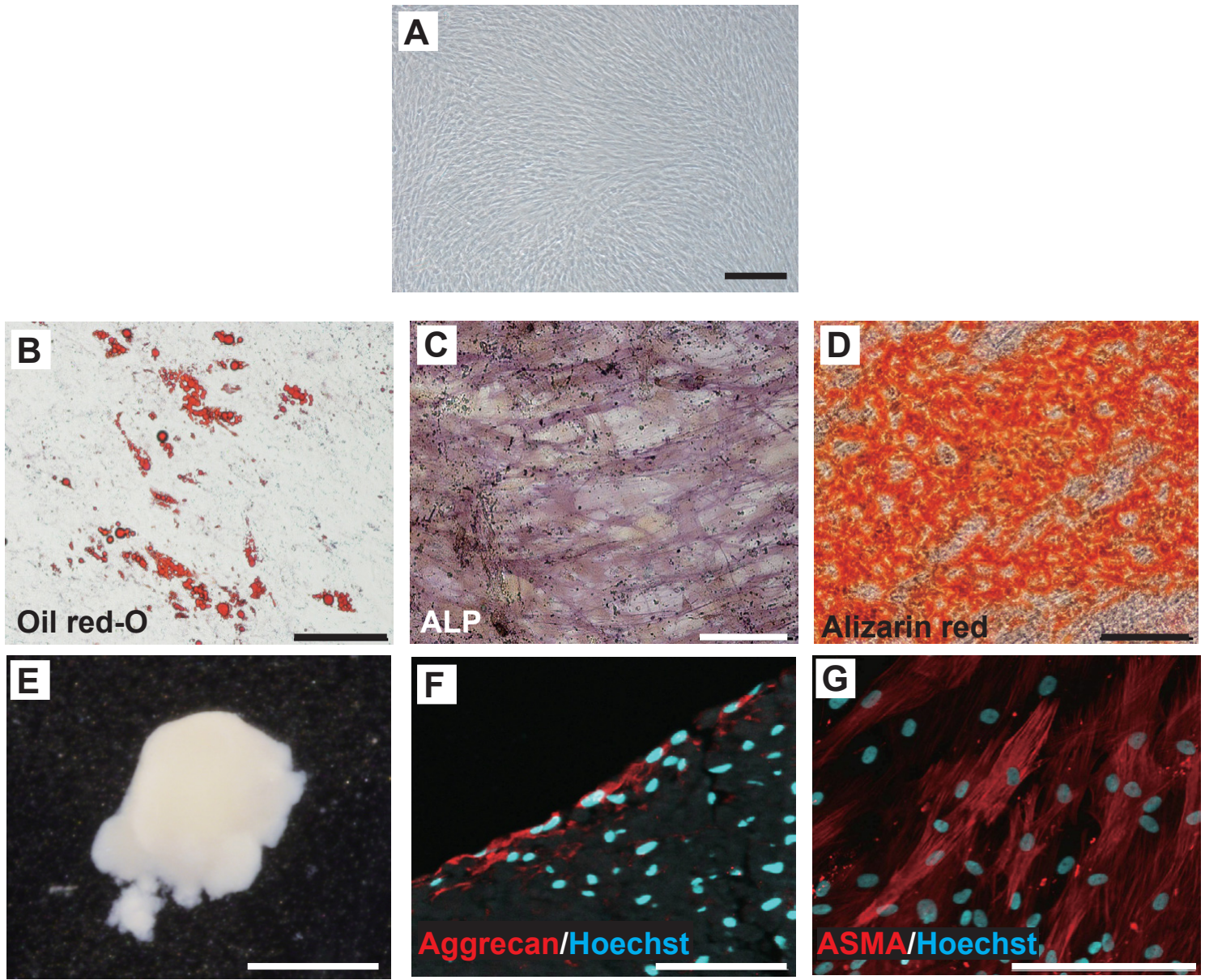

Figure 1: Characteristics of human adipose-derived stem cells (ASCs).

Cultured ASCs were prepared from stromal vascular fraction cells isolated from collagenase-digested human subcutaneous adipose tissue. The cells were analyzed for their multilineage differentiation potential in vitro. (A) Morphology of ASCs at first passage. (B) Cells were cultured in adipogenic differentiation medium for 2 weeks and stained with Oil red $\mathrm{O}$. (C and D) Cells were cultured in osteogenic differentiation medium for 3 weeks and stained for alkaline phosphatase (ALP) (C) and alizarin red $S(D)$. ( $E$ and $F$ ) Cells were seeded in $15-\mathrm{cm}^{3}$ conical tubes and cultured in chondrogenic differentiation medium for 3 weeks. Formed micromass pellets were photographed $(E)$. The micromass pellets were sectioned and stained for aggrecan $(F)$. $(G)$ Cells were cultured in smooth muscle cell differentiation medium for 1 week and stained for a-smooth muscle actin (ASMA). Nuclei were stained with Hoechst 33342 (Hoechst). Scale bar: $200 \mu \mathrm{m}$ in A, B, C, D, F, and G; 1 mm in E. 
Citation: Nakamura T, Kazama T, Nagaoka Y, Inamo Y, Mugishima H, et al. (2015) Influence of Donor Age and Passage Number on Angiogenic Activity in Human Adipose-Derived Stem Cell-Conditioned Media. J Stem Cell Res Ther 5: 307. doi:10.4172/2157-7633.1000307

Page 4 of 10

flattened morphology, although ASCs from donors aged 5 months and 1 year retained their spindle-shaped morphology (Figure 2A). The flattened cells then lost their proliferative potential and could not be subcultured. In ASCs from elderly donors aged more than 60 years, $40 \%$ $(2 / 5)$ and $20 \%(1 / 5)$ of samples could be subcultured up to passages 4 and 6 , respectively, and no samples could be maintained up to passage 8 (Figure 2B). In ASCs from young donors aged 26 years or less, 100\% (5/5) of samples could be subcultured up to passage 6 , and $80 \%(4 / 5)$ of samples could be maintained up to passage 8 (Figure $2 \mathrm{~B}$ ). When the ASC cultures were divided into two groups, the age $\leq 26$ years group and the age $\geq 60$ years group ( $\mathrm{n}=5$ per group), the frequency of cultures that could be passaged up to passage 4 in the age $\geq 60$ years group was significantly lower ( $p<0.05)$ than that in the age $\leq 26$ years group (chi-square test). These results suggest that a donor age over 60 years influenced the proliferative capacity of ASCs at passage 4 and later. Because $60 \%(6 / 10)$ of ASC cultures became senescent by passage 8 , ASC-CM at passages 2 , 4 , and 6 were used for the HUVEC tube formation assay and ELISA to evaluate their angiogenic potential (Figure $2 \mathrm{~B}$, arrows).

\section{ASC passage number affects ASC-CM-induced HUVEC tube formation}

We next examined the effects of ASC-CM on HUVEC tube formation.

A
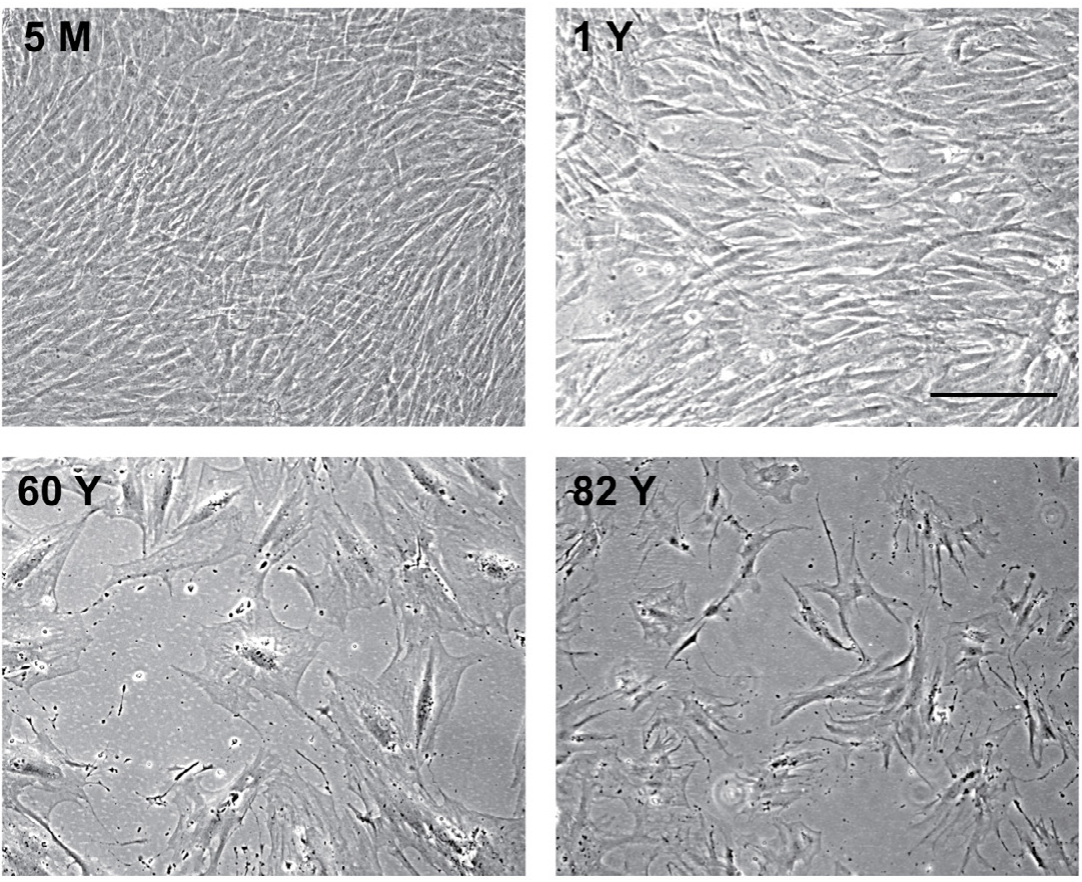

B

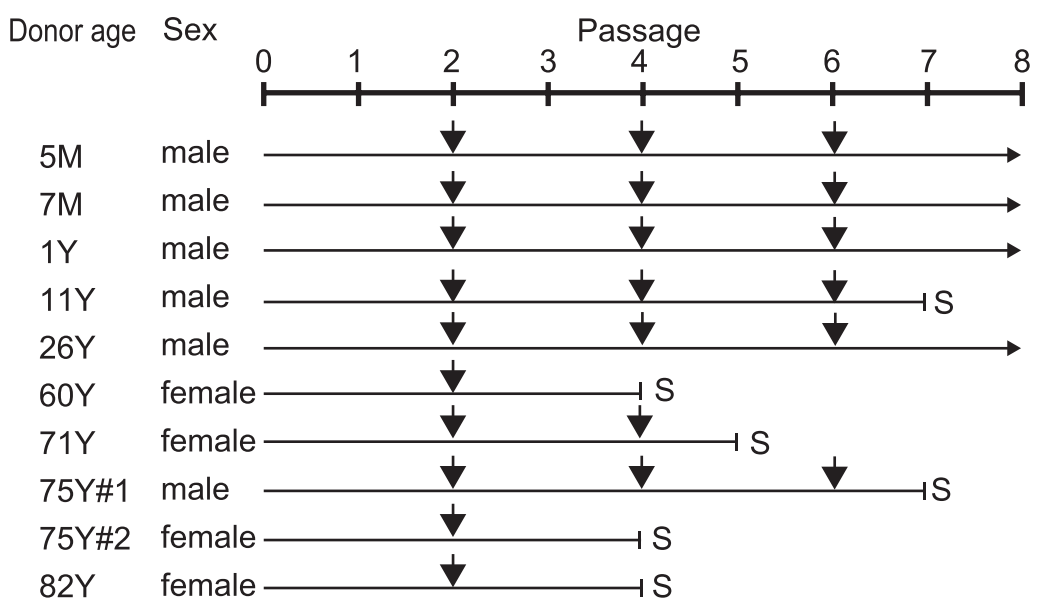

Figure 2: Effect of donor age and passage number on cell morphology and proliferative capacity in cultured ASCs.

(A) Photomicrographs show the morphology of cultured ASCs at passage 4 prepared from donors aged 5 months ( $5 \mathrm{M}), 1$ year $(1 \mathrm{Y}), 60$ years ( $60 \mathrm{Y})$, and 82 years ( 82 Y). Scale bar: $200 \mu \mathrm{m}$. (B) Proliferative capacity of cultured ASCs from each individual during the passaging of cells is shown. $S$ indicates cellular senescence. Arrows indicate periods at which conditioned media were collected for analysis. 
Citation: Nakamura T, Kazama T, Nagaoka Y, Inamo Y, Mugishima H, et al. (2015) Influence of Donor Age and Passage Number on Angiogenic Activity in Human Adipose-Derived Stem Cell-Conditioned Media. J Stem Cell Res Ther 5: 307. doi:10.4172/2157-7633.1000307

Page 5 of 10

We employed a commercially available HUVEC tube formation assay kit in which HUVECs are co-cultured with fibroblasts as feeder cells [32,33]. We confirmed that medium containing VEGF-A induced HUVEC tubelike structures (Figure 3A, PC), whereas unconditioned medium did not induce apparent tube-like structures, especially those with a tube length over $200 \mu \mathrm{m}$ (Figure 3A, NC). Incubation with ASC-CM clearly induced
HUVEC tube-like structures, similar to the VEGF-A-stimulated positive control, regardless of donor age (Figure 3A) and passage number (Figure 3B). Quantitative analysis revealed that the total tube length and total tube area induced by ASC-CM at passages 2 and 4 were equivalent or higher compared to the VEGF-A-stimulated positive control (Figure 4). The actual values for the tube length and tube area varied among ASC-
A
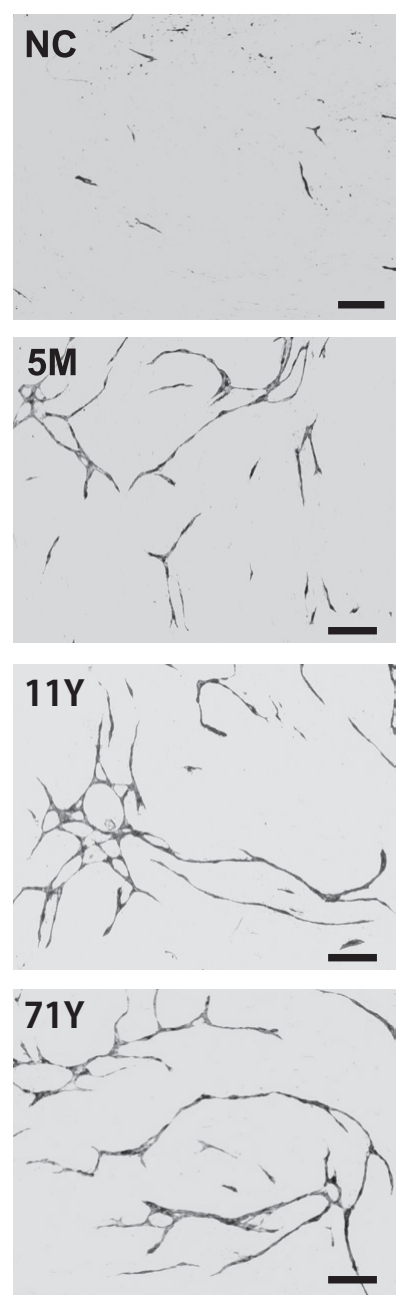

B

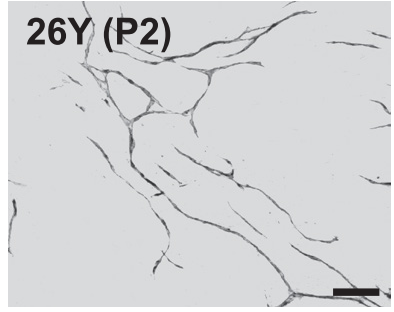

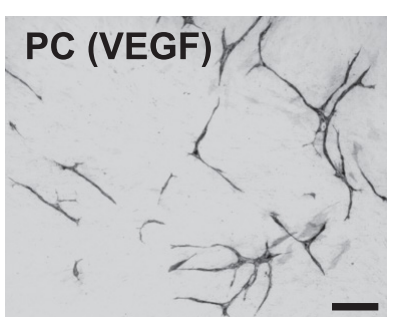
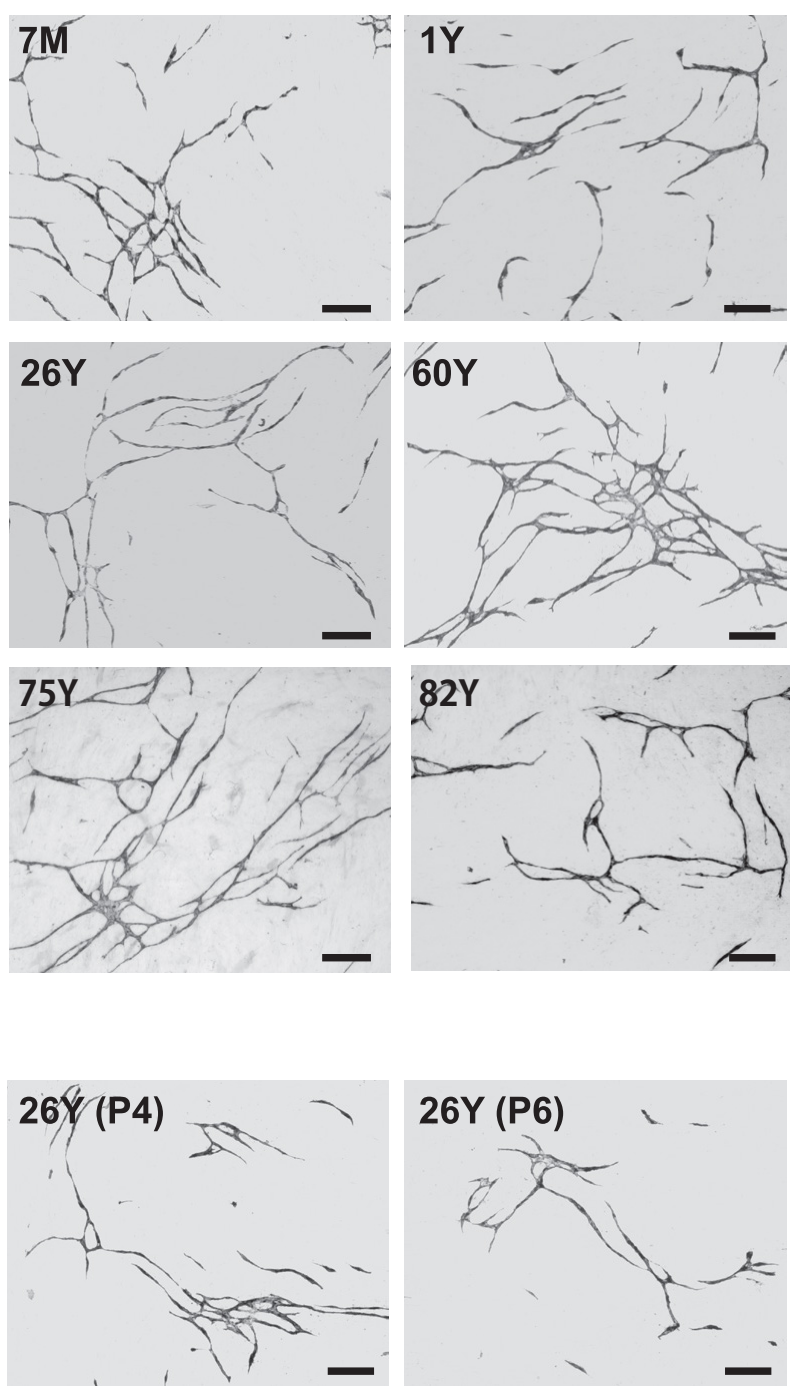

Figure 3: ASC-conditioned media (ASC-CM) enhanced human umbilical vein endothelial cell (HUVEC) tube formation.

Conditioned media were collected from cultured ASCs at passages 2, 4, and 6 from donors of different ages and used in a HUVEC tube formation assay. After 11 days of culture, HUVEC tube-like structures were visualized with CD31 immunostaining and photographed. (A) Representative photomicrographs of HUVEC tube-like structures in cultures treated with ASC-CM from passage 2 from donors aged 5 months $(5 \mathrm{M}), 7$ months (7M), 1 year (1Y), 11 years (11Y), 26 years (26Y), 60 years (60Y), 71 years (71Y), 75 years (75Y), and 82 years ( $82 \mathrm{Y})$. Unconditioned medium was the negative control (NC), and medium containing 10 ng/mI VEGF-A was the positive control (PC). (B) Representative photomicrographs of HUVEC tube-like structures in cultures treated with ASC-CM from passage (P)2, 4, and 6 from donors aged 26 years (26Y). Scale bar: $200 \mu \mathrm{m}$. 

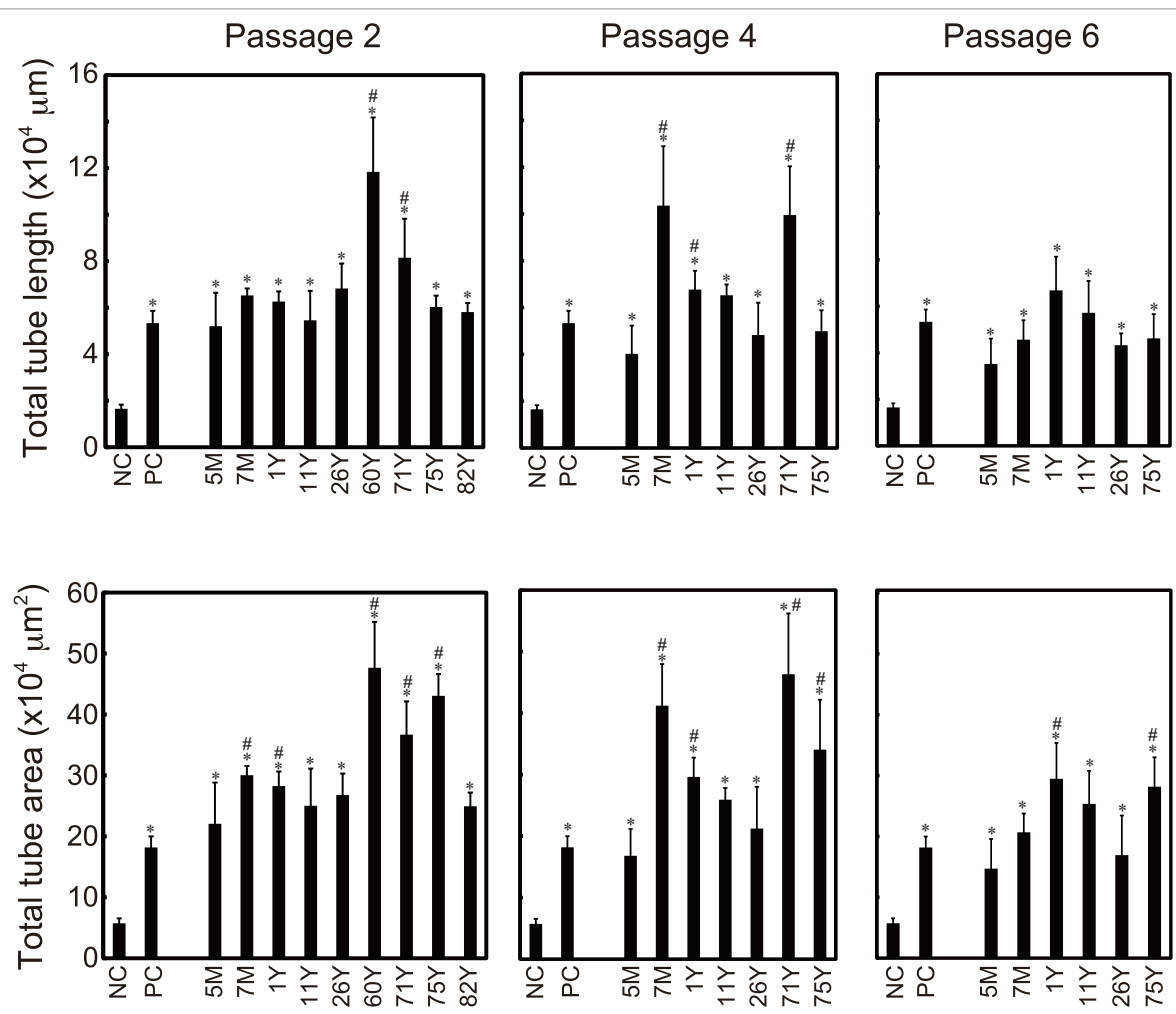

Figure 4: Effect of donor age and passage number on the angiogenic potential of ASC-CM in the HUVEC tube formation assay.

Conditioned media were collected from cultured ASCs at passages 2, 4, and 6 from donors of different ages and used in the HUVEC tube formation assay. After 11 days of culture, HUVEC tube-like structures were visualized with CD31 immunostaining and photographed. Unconditioned medium was the negative control (NC), and medium containing $10 \mathrm{ng} / \mathrm{ml}$ VEGF-A was the positive control (PC). Total tube length and total tube area in three random fields were quantified. Data shown are the mean \pm SD of results from triplicate dishes. " $p<0.05$ vs NC, ${ }^{*} p<0.05$ vs PC (One-way ANOVA and Tukey's multiple comparison test).
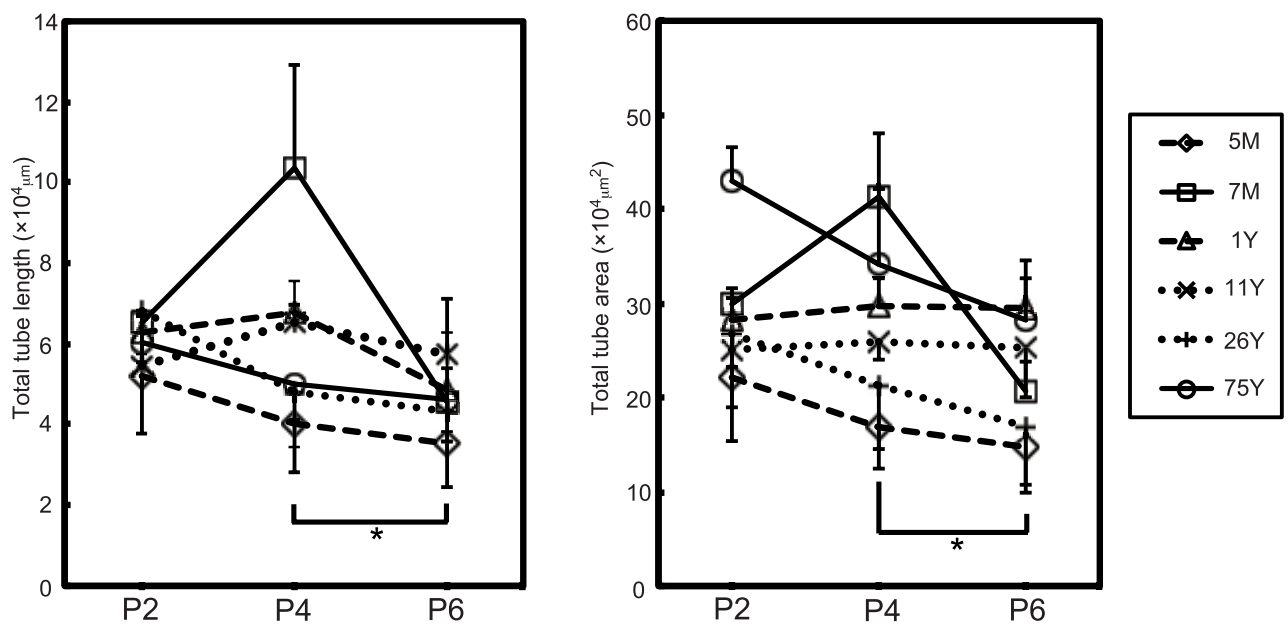

Figure 5: Changes in the tube formation capacity of ASC-CM according to passage number.

Using six donor-derived ASC-CM samples that were serially collected at passages 2, 4, and 6 (P2, P4, and P6), changes in the ASC-CM-induced tube formation capacity were compared for each passage. Data shown are the mean \pm SD of results from triplicate dishes. * $p<0.05$ (Friedman's ANOVA and Wilcoxon signed-rank test).

CM samples, and no clear correlation with donor age was observed. ASC-CM at passage 6 showed a tendency toward a decrease in total tube length and total tube area compared to ASC-CM at passages 2 or 4 , although the values were still equivalent to the positive control (Figure 4). Passage-associated changes in the tube formation capacity of $\mathrm{CM}$ from six donor-derived cultures of ASCs that could be subcultured up to passage 6 are shown in Figure 5. We found that both the tube length and tube area induced by ASC-CM at passage 6 were significantly $(\mathrm{p}<0.05)$ smaller than those induced by ASC-CM at passage 4 (Figure 5). These results suggest that ASC-CM possess significant tube formation capacity regardless of the donor age, although the capacity was decreased when the ASCs reached passage 6. 
A

Passage 2
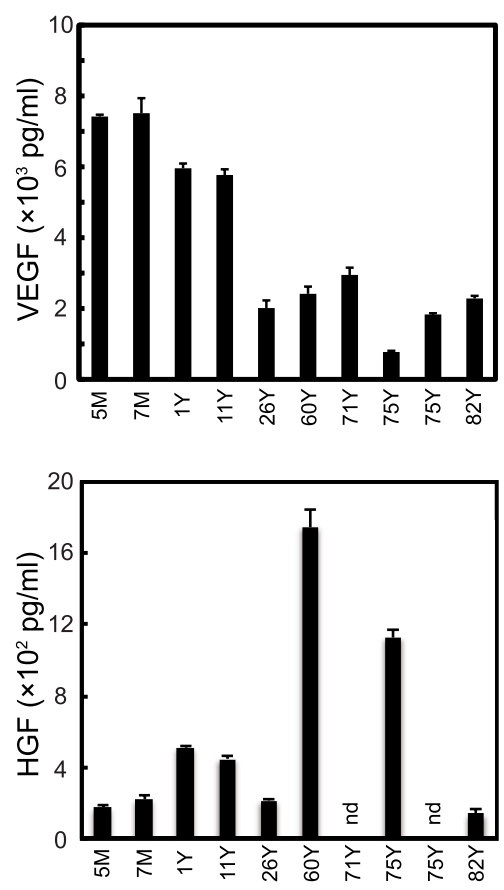

Passage 4
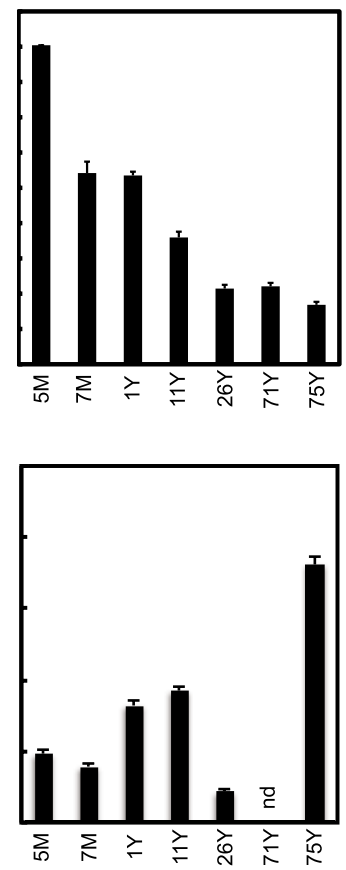

Passage 6
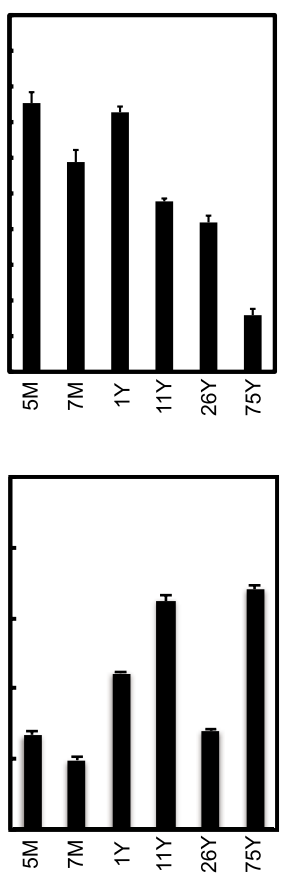

B

Passage 2

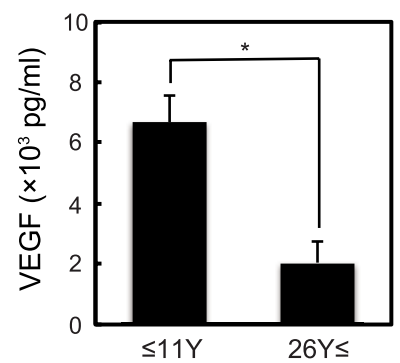

Passage 4

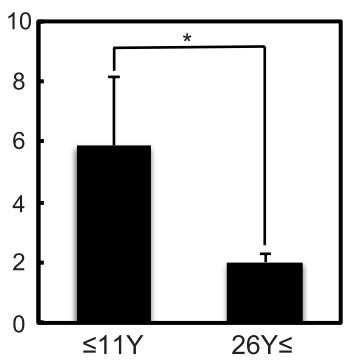

Passage 6

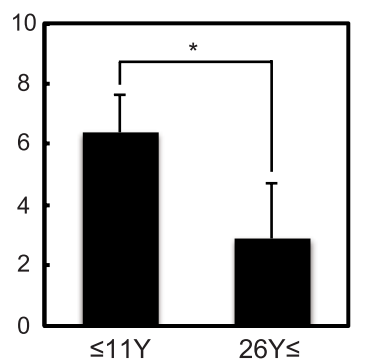

Figure 6: Effect of donor age and passage number on the concentration of angiogenic factors in ASC-CM.

Conditioned media were collected from cultured ASCs at passages 2, 4, and 6 from donors of different ages. The expression levels of vascular endothelial growth factor-A (VEGF-A) and hepatocyte growth factor (HGF) in ASC-CM were measured with ELISA. (A) The concentrations of VEGF-A and HGF in each sample are shown. Data shown are the mean \pm SD of results from triplicate dishes. nd indicates not detectable. (B) The samples were divided into two groups: donor age $\geq 26$ years and donor age $\leq 11$ years. The levels of VEGF-A were compared between the two groups at each passage. Data shown are the mean \pm SD. ${ }^{*} p<0.05$ (Mann-Whitney U test).

\section{Donor age affects VEGF-A secretion by ASCs}

Previous studies demonstrated that angiogenic factors such as VEGF-A [34], FGF-2 [35], and HGF [36] promote HUVEC tube formation. We next evaluated the concentration of these angiogenic factors in ASC-CM using ELISA. Considerable levels of VEGF-A and HGF were detected in ASC-CM in unstimulated conditions (Figure $6 \mathrm{~A}$ ), whereas FGF-2 was undetectable in most samples of ASC-CM $(<10 \mathrm{pg} / \mathrm{mL}$, data not shown). At passage 2, the VEGF-A levels tended to decrease in a donor age-dependent manner (Figure 6A). A similar tendency was observed in ASC-CM at passages 4 and 6 . When the samples were divided into two groups based on the donor age of 26 years as a cut-off point, the VEGF-A levels were significantly lower ( $\mathrm{p}<$ 0.05 ) at passages 2,4 , and 6 in the age $\geq 26$ years group compared to the age $\leq 11$ years group (Figure 6B). On the other hand, HGF levels in ASC-
CM varied greatly among the samples, and we found no correlation between HGF levels and donor age or passage number. Relatively high HGF levels ( $>1 \mathrm{ng} / \mathrm{mL}$ ) were found even in ASC-CM obtained from the oldest donor (75 years old) at the latest passage (passage 6), suggesting that donor age and passage number did not affect HGF secretion by ASCs. Correlation analysis revealed that neither the VEGF-A nor the HGF level correlated with the tube formation capacity in ASC-CM. These results suggest that donor age affects VEGF-A secretion but not HGF secretion in ASCs, and that no significant relationship exists between levels of these angiogenic factors and the tube formation capacity.

\section{Discussion}

In the present study, we used 10 samples from donors ranging 
in age from 5 months to 82 years, and we found that donor age influenced the proliferative capacity of cultured ASCs at passage 4 and later. The results are in agreement with previous studies showing an age-associated decline in proliferative and clonogenic capacities in human bone marrow MSCs [37,38] and human ASCs [24,39,40]. The expression of the senescence-related genes $16^{\mathrm{INK4a}}$ and $\mathrm{CHK} 1$ increases with age in human ASCs [24]. Bone marrow MSCs from patients aged over 40 years show increased levels of reactive oxygen species (ROS) and nitric oxide (NO) and impaired stress responses [37]. In mice, aged ASCs also exhibit increases in ROS and NO production and a decrease in the antioxidative defense enzyme glutathione peroxide with significant telomere shortening [26]. Our study results using 10\% FBS in DMEM as a growth medium imply that ASCs can be cultured until passage 6 for clinical applications. On the other hand, a recent study [41] demonstrated that the age effect on human ASC proliferation and survival was not significant until at least passage 9 when the cells were cultured in K-NAC medium, a modified medium that enhances ASC growth [42]. Optimization of the culture medium may facilitate longterm ASC culture.

With ELISA, we found that the VEGF-A levels in ASC-CM from donors aged $\geq 26$ years were significantly lower than those from donors aged $\leq 11$ years and that the VEGF-A levels were independent of the passage number. These data may result from an increased production of VEGF-A from ASCs in children in the growth period, since VEGF-A accounts for most of the angiogenic activity during adipose tissue development. A similar donor age-dependent decline in VEGF levels has been reported in mouse and rat bone marrow MSCs $[18,43]$ and mouse ASCs [26]. A recent study using human bone marrow MSCs and ASCs demonstrated donor age-associated changes in microRNAs, which regulate expression of several genes and cellular function $[23,24]$. Further studies are needed to identify the essential molecules, including micro-RNAs, that regulate biological age-dependent VEGF-A expression.

Our results showed that the basal secretion level of HGF from ASCs varied and was independent of donor age and passage number. Although ASCs constitutively secrete HGF in vitro [6,44], whether donor age and passage number affect HGF secretion in ASCs is debatable. In mouse ASCs, the level of secreted HGF increases with age despite a decrease in VEGF [26]. In human ASCs, basal levels of HGF in ASCs from donors $>60$ years of age are similar to levels in young ASCs from donors $<35$ years of age, although inducible expression of HGF by macrophage-CM is impaired in ASCs from older donors [45]. In human amnion MSCs, the expression levels of HGF do not change after serial passages, although the levels of VEGF and FGF-2 decrease in a passage number-dependent manner [46]. Further experiments will be required to determine the responsible factors that regulate HGF secretion in ASCs.

Angiogenesis involves the proliferation, migration, and remodeling of endothelial cells during the process of tube formation. HUVECs offer an important in vitro model for studying all three of these processes [47]. The in vitro tube formation assay based on co-culture of HUVECs with fibroblasts provides a more physiological environment in which tubules grow on a matrix naturally laid down by the fibroblasts in the assay, resulting in the formation of capillary tube-like structures [32,33]. In the co-culture assay, our data showed that ASC-CM significantly induced HUVEC tube formation, consistent with previous studies using this assay [8] and the matrigel assay $[26,48]$. We also demonstrated that the ASC-CM-induced tube formation capacity was decreased when the ASC passage number reached passage 6. Interestingly, in contrast to the proliferative capacity data in aged ASCs, the decreased capacity of tube formation was independent of ASC donor age. To our knowledge, this is the first study reporting a passage number-dependent but donor age-independent decline in angiogenic activity in ASC-CM. VEGF-A [34], HGF [36], and FGF-2 [35] stimulate the formation of tube-like structures in this assay system. As shown in Fig. 6, neither VEGF-A nor HGF levels in ASC-CM were influenced by the ASC passage number, at least up to passage 6. In addition, FGF-2 levels in ASC-CM were almost undetectable. These findings imply that other factors may play a role in the passage number-dependent angiogenic activity in ASCCM. Chemokines such as CXCL8 [49] and proteolytic enzymes such as matrix metalloproteinases 3 and 9 [7] stimulate tube formation in HUVECs. Further studies are needed to determine the concentrations of these chemokines/enzymes in ASC-CM and the relationship with passage number.

An unexpected finding was that the donor age of ASCs did not affect ASC-CM-induced tube formation, although VEGF-A levels declined in a donor age-dependent manner. A previous study showed that a linear dose-response relationship exists between the VEGF-A concentration and the tube formation potency in the co-culture assay in which concentrations of $10 \mathrm{ng} / \mathrm{mL}$ VEGF-A and $1 \mathrm{ng} / \mathrm{mL}$ FGF- 2 are required for maximum response [29]. Our data demonstrated that the tube formation capacity of ASC-CM, even at passage 6, was equivalent or higher than that of medium containing $10 \mathrm{ng} / \mathrm{mL}$ VEGF-A as positive control (see Figure 4). These findings suggest that factors other than VEGF-A in ASC-CM compensated adequately for the tube formation potential in the assay. Another possibility is that ASC-CM-derived factors indirectly promoted HUVEC tube formation by stimulating the fibroblasts in the co-culture. A previous study demonstrated that exogenous administration of VEGF does not critically affect HUVEC network formation, whereas fibroblast-derived paracrine factors provide a sufficient level of VEGF that is potentially required for the network formation [50]. The role of fibroblasts in response to ASC-CM during HUVEC tube formation requires further study.

Our study has several limitations. First, the number of donors was relatively small for drawing statistically significant conclusions. In particular, samples from donors aged 26-60 years are lacking. Second, underlying diseases in ASC donors were not considered in the present study. Several studies suggest that the number and functional activities of MSCs may be impaired in cells from patients with diseases such as diabetes and osteoporosis $[27,51,52]$. Third, the angiogenic potential of ASC-CM was evaluated only in the unstimulated condition. Because expression of angiogenic factors is regulated by various stimuli such as hypoxia $[26,44,53]$, mRNA expression and protein secretion of angiogenic factors in primed or stimulated ASCs will need to be examined. Fourth, whether the results from the co-culture assay correlate with the angiogenic potential of ASC-CM in vivo is unclear. The effect of aged ASC transplantation on recovery of blood flow in the ischemic animal model will need to be tested. Aged recipient endothelial cells and stromal cells may influence the angiogenic activity in the case of autologous ASC transplantation.

Our data provide some useful information about ASC-based therapy against ischemic diseases. First, ASCs should be prepared and transplanted within passage 4 , because donor age affects the proliferative capacity of ASCs at passage 4 and later, and the tube formation capacity of ASC-CM is retained at passage 4 but is decreased later. Second, ASCs prepared from elderly patients can be clinically used for therapeutic angiogenesis, because donor age does not affect the tube formation capacity of ASC-CM. Third, it is possible that the expression level of 
Citation: Nakamura T, Kazama T, Nagaoka Y, Inamo Y, Mugishima H, et al. (2015) Influence of Donor Age and Passage Number on Angiogenic Activity in Human Adipose-Derived Stem Cell-Conditioned Media. J Stem Cell Res Ther 5: 307. doi:10.4172/2157-7633.1000307

VEGF-A or HGF in ASC-CM cannot predict the clinical outcome of ASC-based therapy, because no significant relationship exists between levels of these angiogenic factors and the tube formation capacity.

\section{Conclusions}

The donor age influenced the proliferative capacity of cultured ASCs at passage 4 and later. In the HUVEC tube formation assay using a system in which fibroblasts were co-cultured, the donor age did not affect the ASC-CM-induced tube formation capacity, although the passage number (passage 6) decreased the capacity. A donor age over 26 years influenced VEGF-A but not HGF levels in ASC-CM, although no direct correlation between VEGF-A/HGF levels and the tube formation capacity was observed. These data provide insight into possible clinical application of autologous ASCs or ASC-CM therapy in elderly patients with ischemic disease. Autologous transplantation of ASCs for therapeutic angiogenesis in elderly patients may be effective if low passage-number cells can be prepared.

\section{Authors' contribution}

TN, TK and YN contributed to practical experiments and data collection. YI and TS contributed to data analysis and interpretation. HM and TM were involved in the conception and design of the study, manuscript writing, and data analysis and interpretation. All authors read and approved the final manuscript.

\section{Acknowledgements}

This research was supported by JSPS KAKENHI Grant Number 26293170 , by JSPS Program for Creating Start-ups from Advanced Research and Technology (START Program, ST261006IP) and by MEXT-Supported Program for the Strategic Research Foundation at Private Universities (S1411018). The authors gratefully thank Chii Yamamoto, Mika Ishige, and Akiko Tsunemi for their technical support regarding our experiments.

\section{References}

1. Zuk PA, Zhu M, Ashijan P, De Ugarte DA, Huang Jl, et al. (2002) Human adipose tissue is a source of multipotent stem cells. Mol Biol Cell 13: 42794295. [PubMed]

2. Dominici M, Le Blanc K, Mueller I, Slaper-Cortenbach I, Marini F, Krause D, et al. Minimal criteria for defining multipotent mesenchymal stromal cells. The International Society for Cellular Therapy position statement. Cytotherapy. 2006;8:315-7.

3. Schaffler A, Büchler C (2007) Concise review: adipose tissue-derived stromal cells--basic and clinical implications for novel cell-based therapies. Stem Cells 25: 818-827. [PubMed]

4. Miranville A, Heeschen C, Sengenès C, Curat CA, Busse R, et al. (2004) Improvement of postnatal neovascularization by human adipose tissue-derived stem cells. Circulation 110: 349-355. [PubMed]

5. Planat-Benard V, Silvestre JS, Cousin B, André M, Nibbelink M, et al. (2004) Plasticity of human adipose lineage cells toward endothelial cells: physiological and therapeutic perspectives. Circulation 109: 656-663. [PubMed]

6. Rehman J, Traktuev D, Li J, Merfeld-Clauss S, Temm-Grove CJ, et al. (2004) Secretion of angiogenic and antiapoptotic factors by human adipose stromal cells. Circulation 109: 1292-1298. [PubMed]

7. Kim Y, Kim H, Cho H, Bae Y, Suh K, et al. (2007) Direct comparison of human mesenchymal stem cells derived from adipose tissues and bone marrow in mediating neovascularization in response to vascular ischemia. Cell Physiol Biochem 20: 867-876. [PubMed]

8. Nakagami H, Maeda K, Morishita R, Iguchi S, Nishikawa T, Takami Y, et al. Novel autologous cell therapy in ischemic limb disease through growth factor secretion by cultured adipose tissue-derived stromal cells. Arterioscler Thromb Vasc Biol. 2005;25:2542-7.

9. Cai L, Johnstone BH, Cook TG, Tan J, Fishbein MC, Chen PS, et al. IFATS collection: Human adipose tissue-derived stem cells induce angiogenesis and nerve sprouting following myocardial infarction, in conjunction with potent preservation of cardiac function. Stem Cells. 2009;27:230-7.

10. Ning H, Liu G, Lin G, Yang R, Lue TF, et al. (2009) Fibroblast growth factor 2 promotes endothelial differentiation of adipose tissue-derived stem cells. J Sex Med 6: 967-979. [PubMed]
11. Wosnitza M, Hemmrich K, Groger A, Graber S, Pallua N (2007) Plasticity of human adipose stem cells to perform adipogenic and endothelial differentiation. Differentiation 75: 12-23. [PubMed]

12. Nie C, Yang D, Xu J, Si Z, Jin X, et al. (2011) Locally administered adiposederived stem cells accelerate wound healing through differentiation and vasculogenesis. Cell Transplant 20: 205-216. [PubMed]

13. Lee WC, Rubin JP, Marra KG (2006) Regulation of alpha-smooth muscle actin protein expression in adipose-derived stem cells. Cells Tissues Organs 183 80-86. [PubMed]

14. Rodriguez LV, Alfonso Z, Zhang R, Leung J, Wu B and Ignarro LJ. Clonogenic multipotent stem cells in human adipose tissue differentiate into functional smooth muscle cells. Proc Natl Acad Sci U S A. 2006;103:12167-72.

15. Kondo K, Shintani S, Shibata R, Murakami H, Murakami R, et al. (2009) Implantation of adipose-derived regenerative cells enhances ischemia-induced angiogenesis. Arterioscler Thromb Vasc Biol 29: 61-66. [PubMed]

16. li M, Horii M, Yokoyama A, Shoji T, Mifune Y, et al. (2011) Synergistic effect of adipose-derived stem cell therapy and bone marrow progenitor recruitment in ischemic heart. Lab Invest 91: 539-552. [PubMed]

17. Gimble JM, Bunnell BA, Guilak F (2012) Human adipose-derived cells: an update on the transition to clinical translation. Regen Med 7: 225-235. [PubMed]

18. Wilson A, Shehadeh LA, Yu H, Webster KA (2010) Age-related molecular genetic changes of murine bone marrow mesenchymal stem cells. BMC Genomics 11: 229. [PubMed]

19. Wagner W, Horn P, Castoldi M, Diehlmann A, Bork S, et al. (2008) Replicative senescence of mesenchymal stem cells: a continuous and organized process. PLoS One 3: e2213. [PubMed]

20. Kretlow JD, Jin YQ, Liu W, Zhang WJ, Hong TH, et al. (2008) Donor age and cell passage affects differentiation potential of murine bone marrow-derived stem cells. BMC Cell Biol 9: 60. [PubMed]

21. Pan Z, Yang J, Guo C, Shi D, Shen D, et al. (2008) Effects of hindlimb unloading on ex vivo growth and osteogenic/adipogenic potentials of bone marrow-derived mesenchymal stem cells in rats. Stem Cells Dev 17: 795-804. [PubMed]

22. Kim M, Kim C, Choi YS, Kim M, Park C, et al. (2012) Age-related alterations in mesenchymal stem cells related to shift in differentiation from osteogenic to adipogenic potential: implication to age-associated bone diseases and defects. Mech Ageing Dev 133: 215-225. [PubMed]

23. Pandey AC, Semon JA, Kaushal D, O'Sullivan RP, Glowacki J, Gimble JM et al. MicroRNA profiling reveals age-dependent differential expression of nuclear factor kappaB and mitogen-activated protein kinase in adipose and bone marrow-derived human mesenchymal stem cells. Stem Cell Res Ther. 2011;2:49.

24. Alt EU, Senst C, Murthy SN, Slakey DP, Dupin CL, et al. (2012) Aging alters tissue resident mesenchymal stem cell properties. Stem Cell Res 8: 215-225. [PubMed]

25. Zhou S, Greenberger JS, Epperly MW, Goff JP, Adler C, et al. (2008) Agerelated intrinsic changes in human bone-marrow-derived mesenchymal stem cells and their differentiation to osteoblasts. Aging Cell 7: 335-343. [PubMed]

26. Efimenko A, Starostina E, Kalinina N, Stolzing A (2011) Angiogenic properties of aged adipose derived mesenchymal stem cells after hypoxic conditioning. $J$ Transl Med 9: 10. [PubMed]

27. El-Ftesi S, Chang El, Longaker MT, Gurtner GC (2009) Aging and diabetes impair the neovascular potential of adipose-derived stromal cells. Plast Reconstr Surg 123: 475-485. [PubMed]

28. Heydarkhan-Hagvall S, Schenke-Layland K, Yang JQ, Heydarkhan S, Xu Y, et al. (2008) Human adipose stem cells: a potential cell source for cardiovascular tissue engineering. Cells Tissues Organs 187: 263-274. [PubMed]

29. Sarkanen JR, Mannerström M, Vuorenpää H, Uotila J, Ylikomi T, et al. (2011) Intra-Laboratory Pre-Validation of a Human Cell Based in vitro Angiogenesis Assay for Testing Angiogenesis Modulators. Front Pharmacol 1: 147. [PubMed]

30. Matsumoto T, Kano K, Kondo D, Fukuda N, Iribe Y, et al. (2008) Mature adipocyte-derived dedifferentiated fat cells exhibit multilineage potential. J Cell Physiol 215: 210-222. [PubMed]

31. Sakuma T, Matsumoto T, Kano K, Fukuda N, Obinata D, et al. (2009) Mature, adipocyte derived, dedifferentiated fat cells can differentiate into smooth 
Citation: Nakamura T, Kazama T, Nagaoka Y, Inamo Y, Mugishima H, et al. (2015) Influence of Donor Age and Passage Number on Angiogenic Activity in Human Adipose-Derived Stem Cell-Conditioned Media. J Stem Cell Res Ther 5: 307. doi:10.4172/2157-7633.1000307

muscle-like cells and contribute to bladder tissue regeneration. J Urol 182: 355365. [PubMed]

32. Bishop ET, Bell GT, Bloor S, Broom IJ, Hendry NF, et al. (1999) An in vitro model of angiogenesis: basic features. Angiogenesis 3: 335-344. [PubMed]

33. Donovan D, Brown NJ, Bishop ET, Lewis CE (2001) Comparison of three in vitro human 'angiogenesis' assays with capillaries formed in vivo. Angiogenesis 4: 113-121. [PubMed]

34. Okano H, Onmori R, Tomita $\mathrm{N}$ and Ikada $\mathrm{Y}$. Effects of a moderate-intensity static magnetic field on VEGF-A stimulated endothelial capillary tubule formation in vitro. Bioelectromagnetics. 2006;27:628-40.

35. Matou S, Colliec-Jouault S, Galy-Fauroux I, Ratiskol J, Sinquin C, Guezennec $\mathrm{J}$, et al. Effect of an oversulfated exopolysaccharide on angiogenesis induced by fibroblast growth factor-2 or vascular endothelial growth factor in vitro. Biochem Pharmacol. 2005;69:751-9.

36. Saiki A, Watanabe F, Murano T, Miyashita Y, Shirai K (2006) Hepatocyte growth factor secreted by cultured adipocytes promotes tube formation of vascular endothelial cells in vitro. Int J Obes (Lond) 30: 1676-1684. [PubMed]

37. Stolzing A, Jones E, McGonagle D, Scutt A (2008) Age-related changes in human bone marrow-derived mesenchymal stem cells: consequences for cell therapies. Mech Ageing Dev 129: 163-173. [PubMed]

38. Sethe S, Scutt A, Stolzing A (2006) Aging of mesenchymal stem cells. Ageing Res Rev 5: 91-116. [PubMed]

39. Van Harmelen V, Rohrig $\mathrm{K}$ and Hauner $\mathrm{H}$. Comparison of proliferation and differentiation capacity of human adipocyte precursor cells from the omental and subcutaneous adipose tissue depot of obese subjects. Metabolism. 2004;53:632-7.

40. de Girolamo L, Lopa S, Arrigoni E, Sartori MF, Baruffaldi Preis FW and Brini AT. Human adipose-derived stem cells isolated from young and elderly women: their differentiation potential and scaffold interaction during in vitro osteoblastic differentiation. Cytotherapy. 2009;11:793-803.

41. Chen $\mathrm{HT}$, Lee MJ, Chen $\mathrm{CH}$, Chuang SC, Chang LF, Ho ML, et al. Proliferation and differentiation potential of human adipose-derived mesenchymal stem cells isolated from elderly patients with osteoporotic fractures. J Cell Mol Med. 2012;16:582-93

42. Lin TM, Tsai JL, Lin SD, Lai CS, Chang CC (2005) Accelerated growth and prolonged lifespan of adipose tissue-derived human mesenchymal stem cells in a medium using reduced calcium and antioxidants. Stem Cells Dev 14: 92 102. [PubMed]
43. Jiang S, Kh Haider H, Ahmed RP, Idris NM, Salim A, et al. (2008) Transcriptional profiling of young and old mesenchymal stem cells in response to oxygen deprivation and reparability of the infarcted myocardium. J Mol Cell Cardiol 44: 582-596. [PubMed]

44. Kilroy GE, Foster SJ, Wu X, Ruiz J, Sherwood S, et al. (2007) Cytokine profile of human adipose-derived stem cells: expression of angiogenic, hematopoietic, and pro-inflammatory factors. J Cell Physiol 212: 702-709. [PubMed]

45. Scruggs BA, Semon JA, Zhang X, Zhang S, Bowles AC, Pandey AC, et al Age of the donor reduces the ability of human adipose-derived stem cells to alleviate symptoms in the experimental autoimmune encephalomyelitis mouse model. Stem Cells Transl Med. 2013;2:797-807.

46. Fatimah SS, Tan GC, Chua K, Fariha MM, Tan AE, et al. (2013) Stemness and angiogenic gene expression changes of serial-passage human amnion mesenchymal cells. Microvasc Res 86: 21-29. [PubMed]

47. Park HJ, Zhang Y, Georgescu SP, Johnson KL, Kong D and Galper JB. Human umbilical vein endothelial cells and human dermal microvascular endothelia cells offer new insights into the relationship between lipid metabolism and angiogenesis. Stem cell Rev. 2006;2:93-102.

48. Deveza L, Choi J, Imanbayev G, Yang F (2013) Paracrine release from nonviral engineered adipose-derived stem cells promotes endothelial cell survival and migration in vitro. Stem Cells Dev 22: 483-491. [PubMed]

49. Matsuo Y, Ochi N, Sawai H, Yasuda A, Takahashi H, et al. (2009) CXCL8/ IL-8 and CXCL12/SDF-1alpha co-operatively promote invasiveness and angiogenesis in pancreatic cancer. Int J Cancer 124: 853-861. [PubMed]

50. Kunz-Schughart LA, Schroeder JA, Wondrak M, van Rey F, Lehle K, et al. (2006) Potential of fibroblasts to regulate the formation of three-dimensional vessel-like structures from endothelial cells in vitro. Am J Physiol Cell Physiol 290: C1385-1398. [PubMed]

51. Dimmeler S, Leri A (2008) Aging and disease as modifiers of efficacy of cell therapy. Circ Res 102: 1319-1330. [PubMed]

52. Pino AM, Rosen CJ, Rodríguez JP (2012) In osteoporosis, differentiation of mesenchymal stem cells (MSCs) improves bone marrow adipogenesis. Biol Res 45: 279-287. [PubMed]

53. Thangarajah H, Vial IN, Chang E, El-Ftesi S, Januszyk M, et al. (2009) IFATS collection: Adipose stromal cells adopt a proangiogenic phenotype under the influence of hypoxia. Stem Cells 27: 266-274. [PubMed] 